\title{
SUR LA NUTRITION ARTIFICIELLE DES TIQUES
}

\author{
Par A.-G. CHABAUD
}

Des travaux récents montrent l'intérêt des méthodes de nutrition artificielle des arthropodes piqueurs, aussi bien dans l'étude de la physiologie du parasite que dans les expériences de transmission de germes.

Deux méthodes sont employées. La première, qui consiste à nourrir les animaux à travers une membrane, a été mise parfaitement au point par P. Nicolle (1941 et 1944) pour les réduvidés hémophages. Elle est employée fréquemment pour les moustiques (cf. en particulier les travaux récents et la bibliographie de Bishop et Gilchrist, 1946). Des expériences basées sur les mêmes principes avaient été faites par Hindle et Duncan (1925) pour Argas persicus, puis par Totze (1933 et 1934) pour les ixodes.

La seconde méthode est une application des travaux de Fülleborn (1908). Elle permet de faire absorber un liquide à l'animal par l'introduction des pièces buccales dans un tube capillaire. Ce procédé a donné d'excellents résultats pour les moustiques (Mac Gregor 1930, Fülleborn 1932) et pour les phlébotomes (Hertig et Hertig 1927), mais n'a pas encore à notre connaissance été utilisé pour les tiques.

A côté de ces deux techniques, Blanc et Baltazard (1944) infectent des puces de façon moins artificielle. Ils créent une bactériémie intense, cinq minutes avant le repas, en injectant à l'hôte par voie intraveineuse une solution suffisamment concentrée des germes expérimentés.

Le grand inconvénient présenté par les méthodes de nutrition à travers membrane est la nécessité d'un appareillage relativement compliqué. Au contraire, pour l'alimentation forcée par tube capillaire, il est toujours facile d'improviser une pipette à double effilure et cela ne nécessite qu'une très petite quantité du liquide à expérimenter.

Chez les tiques, le succès des nutritions à travers membrane dépend essentiellement de deux facteurs : d'une part, la neutralisa-

Ann. de Parasitologie, $\mathrm{T}$. XXV, $\mathrm{N}^{\circ \times} 1-2 .-1950$, p. 42-47. 
tion des organes de Haller ; d'autre part, le réglage à une température convenable du liquide qui est offert. Nous n'avons pas su mettre en évidence les facteurs qui déterminent l'absorption de liquide par les tiques nourries avec un tube capillaire. Les résultats n'ont été améliorés ni par l'ablation des organes de Haller, ni par le réchauffement du liquide (obtenu par différents procédés : faisceau lumineux concentré par une loupe, plaque chauffante, etc...). Nous n'avons donc pu obtenir par ce procédé que des résultats partiels ou inconstants.

Il est cependant difficile de comparer ces résultats avec ceux qui peuvent être obtenus par l'alimentation à travers une membrane. Totze ne signale nulle part, dans ses deux mémoires, l'obtention d'une mue (pour une nymphe) ou d'une ponte (pour une femelle) consécutives à un repas artificiel ; cela nous parait pourtant être le seul test d'une nutrition convenable. De même, Chorine et Colas Belcour (1947), qui ont utilisé un procédé adapté de celui de Nicolle, n'ont pu nourrir Ornithodorus erraticus que de façon partielle. Quoi qu'il en soit, et même dans le cas où la nutrition obtenue serait moins complète, nous croyons bon de décrire cette seconde méthode qui nous paraît utile par son extrême simplicité.

Technique. - Le procédé le plus facile pour immobiliser l'animal sans traumatisme est de le maintenir par de la paraffine.

On dépose une goutte de paraffine chaude sur une lame porteobjet tiède, puis la lame est retournée d'un mouvement brusque pour obtenir une goutte pendante. On attend alors que les bords de la goutte de paraffine commencent à se solidifier et, à ce moment seulement, on l'applique rapidement sur la partie postérieure de la face dorsale de la tique. On maintient ensuite, pendant quelques secondes, la tique immobile en la pressant entre la lame et la surface sur laquelle elle est posée. Il est alors possible de retourner la lame et de compléter l'immobilisation en fixant les pattes. Chaque patte est étendue à l'aide d'une pince fine pour être fixée par une très petite goutte de paraffine tiède, déposée au niveau du protarse. La contention des trois paires de pattes postérieures suffit à donner une bonne fixation, et généralement nous laissons libre la première paire pour respecter l'organe de Haller (cette précaution paraît d'ailleurs être superflue, car certains spécimens ayant la première paire immobilisée se sont gorgés de façon sensiblement normale).

Le second temps de l'opération consiste à introduire le rostre de la tique dans une effilure de pipette aussi fine que possible. Il y a avantage à déposer au préalable sur la lame une grosse goutte de paraffine, dont le dessus creusé en gouttière servira à maintenir le 


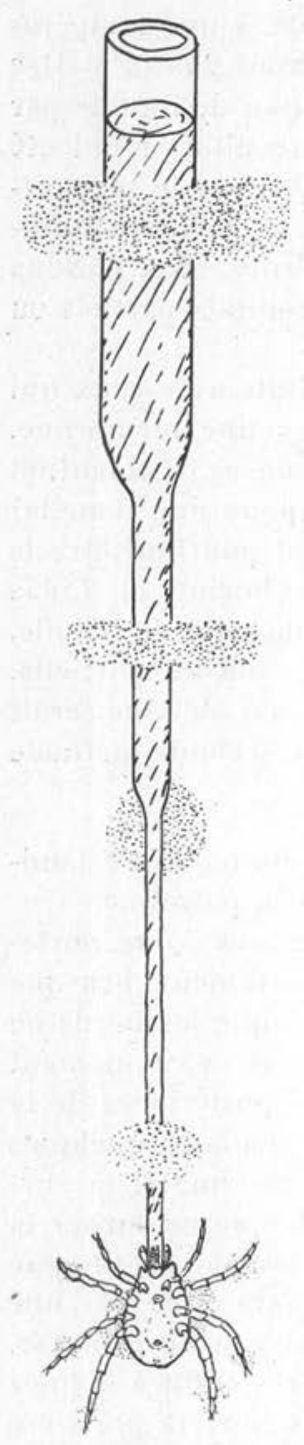

Fig. - Fixation du tube capillaire et de la tique sur une lame porte - objet. (Les zones pointillées indiquent la place des attaches de paraffine). tube dans la position voulue. Un choix de pipettes à double effilure (Langeron, 1949, p. 323) ayant été préparé à l'avance, il faut pousser sous la loupe binoculaire une pipette de taille convenable, jusqu'à ce qu'elle entoure complètement le rostre. Les palpes sont rejetées latéralement sans difficulté, les chélicères et leur gaine peuvent être introduits dans la pipette, mais il semble qu'on obtienne de meilleurs résultats en les repoussant en arrière et en ne laissant que l'hypostome dans l'effilure de la pipette.

Le tube est alors fixé dans sa position définitive par deux ou trois anneaux de paraffine et rempli de liquide à l'aide d'une pipette bien effilée pour éviter l'introduction de bulles d'air. La préparation est placée dans une étuve à $27^{\circ}$, en milieu humide.

Pour les Argasidæ, la fixation de la partie dorsale du corps suffit en général à immobiliser l'animal, et les pattes peuvent être laissées libres. L'introduction de l'hypostome dans la pipette est facile pour certaines espèces comme Ornithodorus moubata ou $O$ parkeri, où le rostre est bien dégagé. Par contre, chez $O$. erraticus ou $O$. talaje, le rostre est enfoncé en profondeur, plus ou moins recouvert par les joues du camérostome, et nous n'avons pu obtenir aucun résultats avec ces espèces.

Expérimentation. - a. Ixodidæ. - Nous avons utilisé avec succès les individus adultes des espèces suivantes : Hyalomma excavatum (Koch), H. dromedarii (Koch), Dermacentor pictus (Herm.) et Rhipicephalus sanguineus Latr. Quelle que soit la nourriture offerte (eau ordinaire, eau physiologique, sérum de cobaye, liquide d'ascite de souris ou sang défibriné de cobaye), les résultats sont sensiblement les mêmes. 
Les mâles se gorgent en 12 ou 18 heures et atteignent un volume comparable à celui des individus nourris de façon normale. Les femelles, après un séjour de 18 à 48 heures dans l'étuve, sont gorgées de façon variable, atteignant souvent le volume d'un petit pois. Le repas s'arrête toujours à ce moment et il a été impossible d'obtenir des individus normalement gorgés. Ce résultat s'explique probablement par l'existence de la diapause trophique virginale (cf. E. Brumpt, 1949, p. 1079).

Nous n'avons pas pu obtenir de fécondation in vitro. Les mâles de Rhipicéphales nourris (artificiellement avec du sérum ou naturellement sur un chien), mis en contact avec des femelles semi-gorgées, n'ont manifesté aucun instinct sexuel.

L'alimentation forcée a été tentée d'autre part sur deux femelles d'Ixodes hexagonus Leach, qui avaient été observées in copula alors qu'elles étaient encore à jeun ; les deux spécimens n'ont absorbé qu'un très petit volume du liquide qui leur était offert (eau physiologique pour la première et sérum pour la seconde) et n'ont pas pondu.

Le transit intestinal paraît dans ces conditions être toujours très rapide. Lorsque les animaux absorbent de l'eau physiologique, on observe très vite un dépôt de sel autour de l'anus. Pour le sang défibriné, il y a émission, par l'anus, d'hémoglobine rouge, deux ou trois heures après le début du repas.

b. Argasidx. - Alors que les Ixodidx se gorgent toujours plus ou moins, les Argasidæ au contraire refusent très souvent le repas. Dans un certain pourcentage de cas cependant, ils acceptent une bonne quantité de nourriture et peuvent même atteindre en quelques minutes un volume comparable à celui des exemplaires gorgés sur animaux. Nous n'avons pas encore pu obtenir de mue à la suite de repas artificiel.

Une nymphe d'O. moubata (Murray), bien gorgée avec du sérum de cobaye le $1^{\text {er }}$ juin 1948, est restée jusqu'au 21 octobre sans muer ; nourrie à ce moment sur un cobaye, elle a mué quelques jours après et a continué son évolution de façon normale. Il est donc possible d'infecter de façon massive des Ornithodores avec un germe quelconque et d'effectuer par la suite des expériences de transmission par piqûre.

Exemple d'application pratique : Les Arthropodes piqueurs, nourris sur des animaux toxoplasmiques même très infectés, ne puisent qu'un très petit nombre de toxoplasmes. Ceux-ci peuvent être décelés par une inoculation immédiate du broyat du vecteur à un verté- 
bré sensible, mais les toxoplasmes sont toujours beaucoup trop rares pour pouvoir être examinés sur un frottis.

A l'Institut Pasteur du Maroc, avec le matériel mis à notre disposition par le $\mathrm{D}^{\mathrm{r}} \mathrm{G}$. Blanc, nous avons fait gorger partiellement des femelles de Rhipicephalus sanguineus avec le liquide d'ascite de souris extrêmement riche en toxoplasmes. Ceux-ci disparaissent en quelques heures du tube digestif de la tique ; nous n'avons pas noté de modification morphologique qui puisse faire penser à un début d'évolution dans ce milieu.

\section{RÉSUMÉ}

Nous proposons une méthode très simple pour nourrir artificiellement les tiques. Il est possible de faire gorger les mâles et partiellement les femelles d'Ixodidæ. Les Argasidæ n'acceptent les repas que de façon inconstante et, même dans les cas les plus favorables, la quantité de liquide absorbé n'a pas suffi à assurer la mue. Malgré ces imperfections, nous pensons que la méthode peut rendre de réels services dans des expériences de physiologie ou de transmission de germes.

\section{Bibliographie}

Bishop (A.) et Gilchrist (B. M.). - Experiments upon the feeding of Aedes agypti through animal membranes with a view to applying this method to the chemotherapy of malaria. Parasitology, XXXVII, 1946, 85-100.

Blanc (G.) et Bat.tazard (M.). - Contribution à l'étude du comportement des microbes pathogènes chez les insectes hématophages. Premier mémoire. Arch. Inst. Past. Maroc, III, 1944, 31-50.

Brumpt (E.). - Précis de Parasitologie, 6édition, 1949, Paris, Masson et Cie.

Chorine (V.) et Colas-Belcour (J.). - Perte du pouvoir infectant des cultures de Spirochata hispantca pour l'Ornithodorıs erraticus, son vecteur dans la nature. Bull. Soc. path. exot., XL, 1947, 383-383.

FülleborN (F.). - Ueber Versuche an Hundefilarien und deren Ubertragung durch Müchen. Arch. Schiffs. Trop. Hyg., XII, 1908, Beineft. 8, 43 p. + 4 pl.

-- Ueber den Saugakt der Stechmücken. Arch. Schiffs. Trop. Hyg., XXXVI, $1932,169-181$.

Hertig (A. T.) et Herisg (M.). - A technique for the artificial feeding of sandflies (Phlebotomus and mosquitoes). Science, LXV, 1927, 328-329.

Hindle (E.) et Duncan (J. T.). - The viability of Bacteria in Argas persicus. Parasitology, XVII, 1925, 434-448 + 1 pl.

Langeron (M.). - Précis de Microscopie, $7^{\circ}$ édition, 1949, Paris, Masson et Cie. 
MAC GREGor (M. E.). - The artificial feeding of mosquitoes by a new method which demonstrates certain functions of the diverticula. Trans. Roy. Soc. Trop. Med. Hyg., XXIII, 1930, 329-331.

Nicolle (P.). - Appareil pour l'alimentation artificielle des réduvidés hémophages. Bull. Soc. path. exot., XXXIV, 1941, 179-185.

- Dispositif permettant la réalisation facile des transmissions infectieuses par voie orale chez les réduvidés hémophages. Bull. Soc. path. exot., XXXVII, 1944, 238-240.

Totze (R.). - Beiträge zur Sinnesphysiologie der Zecken. Zeitsch. f. verglei. Physio., XIX, 1933, 110-161.

Blutsaugen unter rein experimentellen Bedingungen. Zentr. Bakt., CXXXII, 1934, 382-384.

Institut de parasitologie de la Faculté de Médecine de Paris (Directeur : Prof. H. Galliard). 